\title{
Rhabdomyolysis Associated with Fenofibrate Monotherapy in a Patient with Chronic Myelogenous Leukemia
}

\author{
Kazuya Kato ${ }^{a}$ Astushi Nagase $^{b}$ Minoru Matsuda $^{c}$ \\ Yurina Kato $^{\mathrm{a}}$ Kazuhiko Onodera $^{\mathrm{d}}$ Takako Kawakami $^{\mathrm{a}}$ \\ Mineko Higuchi $^{\mathrm{a}}$ Yoshiaki Iwasaki ${ }^{\mathrm{e}}$ Masahiko Taniguchi ${ }^{f}$ \\ Hiroyuki Furukawa
}

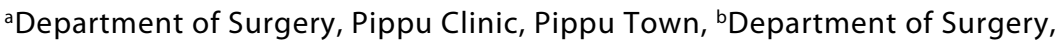
Asahikawa Medical Center, Asahikawa, 'Department of Surgery, Nihon University, Tokyo, ${ }^{d}$ Department of Surgery, Hokuyu Hospital, Sapporo, e Department of Internal Medicine, Okayama University, Okayama, and fDepartment of Surgery, Asahikawa Medical College, Asahikawa, Japan

\section{Key Words}

Rhabdomyolysis · Fenofibrate monotherapy · Chronic myelogenous leukemia · Psoas muscle $\cdot$ Surgery

\begin{abstract}
Rhabdomyolysis associated with fenofibrate monotherapy is extremely rare. Here, we report a rare case of rhabdomyolysis of the psoas muscle in an 82-year-old man with chronic myelogenous leukemia (CML). He was prescribed fenofibrate because of a hypertriglyceridemia. The patient reported generalized muscle pain and right abdominal pain while receiving fenofibrate monotherapy. An abdominal computed tomography scan and an abdominal ultrasound showed a large and low attenuation and high echogenicity, respectively, in the right middle abdominal area. Laboratory values included a serum creatine concentration of $4.1 \mathrm{mg} / \mathrm{dl}$ and a creatinine phosphokinase concentration of 5,882 IU/l. During laparotomy, a large hematoma and necrotic mass was identified in the right psoas muscle. Histological examination revealed that the resected specimens were of the psoas muscle with irregular fiber sizes, degenerating fibers surrounding the inflammatory reaction, and fiber necrosis that is typical for polymyositis. Based on these findings and the clinical history, a diagnosis of fenofibrate-induced rhabdomyolysis was made. To the best of our knowledge, no patient has ever been diagnosed with fulminant psoas rhabdomyolysis due to a fenofibrate monotherapy. This
\end{abstract}

Kazuya Kato, MD, PhD 
report details the rare case of rhabdomyolysis in a patient with CML associated with fenofibrate monotherapy and offers a review of the literature.

\section{Introduction}

Fibric acid agents have long shown their benefit in the treatment of hyperlipidemia. Fenofibrate is effective in reducing serum LDL cholesterol and triglyceride concentrations. It is frequently used alone or in combination with statins to treat diabetic dyslipidemia and hypertriglyceridemia. The most important side effect of fibrates is rhabdomyolysis. Statins are also associated with rhabdomyolysis, and perhaps this raises concerns about possible adverse drug interactions. Rhabdomyolysis is a clinical and biochemical syndrome that results from skeletal muscle injury and involves the subsequent release of muscle cell constituents into the circulation. The typical clinical presentation includes muscle weakness, myalgias and dark-colored urine due to myoglobinuria, and the diagnosis is usually based on elevated serum skeletal muscle enzyme levels [1].

Rhabdomyolysis associated with fenofibrate monotherapy is extremely rare and may result in myoglobinuria with acute renal failure. Fenofibrate monotherapy is associated with the potentially fatal side effect of rhabdomyolysis, which induces acute renal failure. We report a rare case of fulminant rhabdomyolysis of the psoas muscle with fenofibrate monotherapy in an 82-year-old man with chronic myelogenous leukemia (CML). To the best of our knowledge, there are no reports of fenofibrate monotherapy leading to fulminant psoas rhabdomyolysis in a patient with CML. We report this rare case and review the related literature.

\section{Case Report}

The patient was an 82-year-old man in the chronic phase of CML who also had hypertriglyceridemia. His past medical history was significant for imatinib mesylate (Gleevec $\left.{ }^{\mathrm{TM}}\right)$ treatment $400 \mathrm{mg}$ daily for 4 years since his initial presentation. He had tolerated the medication well and achieved a complete cytogenetic remission. Then imatinib was stopped because of liver dysfunction. Six months later, he was started on $100 \mathrm{mg}$ of fenofibrate daily, 4 months before presentation, to treat hypertriglyceridemia (serum triglycerides: $380 \mathrm{mg} / \mathrm{dl}$ ). Before the initiation of fenofibrate therapy, the patient's serum creatinine was $1.3 \mathrm{mg} / \mathrm{dl}$ and the thyroid-stimulating hormone concentration and liver function were within normal limits. Past medical history contained the following pertinent negatives: no recent viral illness, history of trauma, or epilepsy, and the absence of any other medications that could potentially be associated with rhabdomyolysis.

Upon admission, the patient had generalized muscle pain and right abdominal pain and the following laboratory values: white blood cell count, 186,400/ $\mu \mathrm{l}$; aspartate aminotransferase (AST), 1,677 U/l; alanine aminotransferase (ALT), $619 \mathrm{U} / \mathrm{l} ; \mathrm{LDH}, 3,380 \mathrm{U} / \mathrm{l}$; triglycerides, $234 \mathrm{mg} / \mathrm{dl}$; total cholesterol, $117 \mathrm{mg} / \mathrm{dl}$; myoglobin, $990 \mu \mathrm{g} / \mathrm{ml}$; creatine, $4.1 \mathrm{mg} / \mathrm{dl}$; and creatinine phosphokinase (CPK), $5,882 \mathrm{IU} / \mathrm{l}$. The red blood cell and platelet counts were within normal limits. The elevated CPK was not cardiac in origin because both electrocardiography and myocardial enzyme markers were within normal limits. An abdominal computed tomography (CT) scan and ultrasonography showed a large and low attenuation and high echogenicity, respectively, in the right middle retroperitoneal area (fig. 1a). No calcification was observed in the psoas muscle (fig. 1b). During a subsequent laparotomy, a large hematoma and necrotic mass was found in the right psoas muscle (fig. $2 a, b)$. Histological examination of the resected specimens revealed a psoas muscle with disproportionate fiber sizes and degenerating fibers surrounding the inflammatory reaction. These findings, along with the clinical history, confirmed 
a diagnosis of fenofibrate-induced rhabdomyolysis (fig. $3 \mathrm{a}: \times 40$, b: $\times 200$ ). The patient died 3 days after the operation due to acute renal failure.

\section{Discussion}

Fibric acid agents have long shown their benefit in the treatment of hyperlipidemia. Fenofibrate is effective in reducing serum LDL cholesterol and triglyceride concentrations $[2,3]$. It has been shown to increase the serum concentration of HDL cholesterol and reduce the serum concentration of dense LDL cholesterol. Fenofibrate in combination with statins is being used more frequently for the treatment of combined hyperlipidemia and to lower non-HDL cholesterol. Statins are also associated with rhabdomyolysis, and perhaps this raises concerns about possible adverse drug interactions. The side effects of fibrate treatment include gastrointestinal complaints, gallstones, and skin reactions, all of which are tolerable and reversible. The most important side effect of fibrates is rhabdomyolysis $[4,5]$. All fibrates, either as monotherapy or in combination with statins or other agents, are associated with rhabdomyolysis. Gemfibrozil had the highest reported rates of rhabdomyolysis, followed by bezafibrate, fenofibrate, ciprofibrate, and clofibrate [6]. Fibrates are most commonly combined with cerivastatin [6]. Rhabdomyolysis associated with fenofibrate monotherapy is extremely rare. The clinical manifestations of rhabdomyolysis associated with fibrates are nonspecific. This condition presents as myalgias, weakness, fatigue, and dark-colored urine, which usually develop within a few days of starting the treatment. Rhabdomyolysis associated with fibrates appears 3 weeks to 3 months after the initiation of the medication [5]. The mechanism of rhabdomyolysis associated with fibrate therapy remains unclear. It has been suggested that fibrates cause a cell-specific injury to human embryonal rhabdomyosarcoma cells in vitro via the activation of the nuclear receptor peroxisome proliferator-activated receptor-alpha, through which the lipid-lowering action of fibrates is facilitated [7]. It was hypothesized that fibrates only exacerbate latent preexisting mitochondrial myopathies or accelerate the normal physiologic changes in skeletal muscle [8]. We have reported a case of fulminant rhabdomyolysis of the psoas muscle in an 82-year-old man with chronic myelogenous leukemia (CML). The rhabdomyolysis of the psoas iliac muscle was detected using Tc-99m HMDP bone scintigraphy [9]. A case of fulminant rhabdomyolysis of the psoas muscle, which needed an operation, has not previously been reported.

Myalgias and muscle complaints are common side effects in patients who take imatinib for CML [10]. Elevations of CPK are described in only $0.1-1 \%$ of patients, according to the manufacturer's labeling. Cases of muscle edema and rhabdomyolysis have rarely been reported $[11,12]$. These are the etiologic mechanisms of imatinib-induced CPK elevation, and occur early in the course of treatment [13]. The elevated CPK levels normalized within a few weeks after stopping imatinib [10]. The patient in the current report had tolerated the medication well and was in complete cytogenetic remission. Imatinib was stopped because of liver dysfunction 6 months before the presentation of rhabdomyolysis. Oliguric or nonoliguric acute renal failure is the most common complication of rhabdomyolysis, occurring in $10-40 \%$ of patients [14]. Acute renal failure secondary to fenofibrate monotherapy-induced rhabdomyolysis is rare [15].

The current report describes a rare case of rhabdomyolysis in a patient with CML associated with fenofibrate monotherapy. Fenofibrate monotherapy is associated with the potentially fatal side effect of rhabdomyolysis, which induces acute renal failure. To the 


\begin{tabular}{r|l|l|l}
$\begin{array}{r}\text { Case Reports in } \\
\text { Gastroenterology }\end{array}$ & $\begin{array}{l}\text { Case Rep Gastroenterol 2011;5:492-496 } \\
\text { DOl: 10.1159/000331559 }\end{array}$ & $\begin{array}{l}\text { Published online: } \\
\text { August 27, 2011 }\end{array}$ & $\begin{array}{l}\text { O 2011 S. Karger AG, Basel } \\
\text { ISSN 1662-0631 } \\
\text { www.karger.com/crg }\end{array}$ \\
\hline
\end{tabular}

best of our knowledge, this is the first report of fenofibrate monotherapy resulting in fulminant rhabdomyolysis of the psoas muscle.

\section{Disclosure Statement}

The authors declare no conflict of interest.
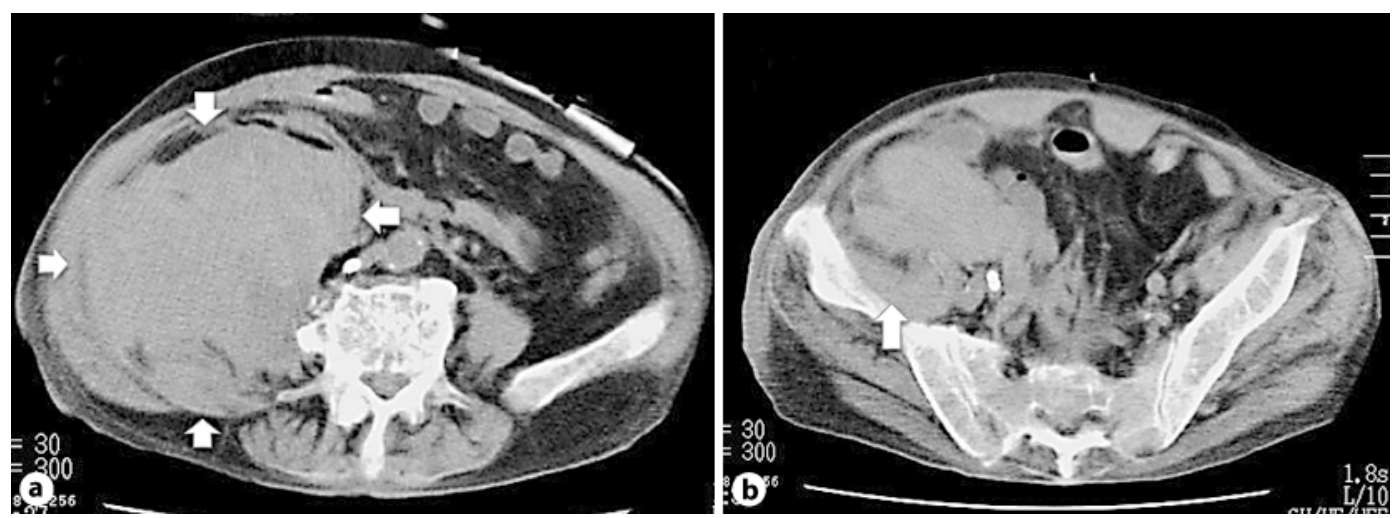

Fig. 1. a An abdominal CT scan showing a large area of low attenuation in the psoas muscle (arrows). b The iliacus muscle was slightly edematous. No calcification was observed in either the psoas muscle or the iliacus muscle (arrow).
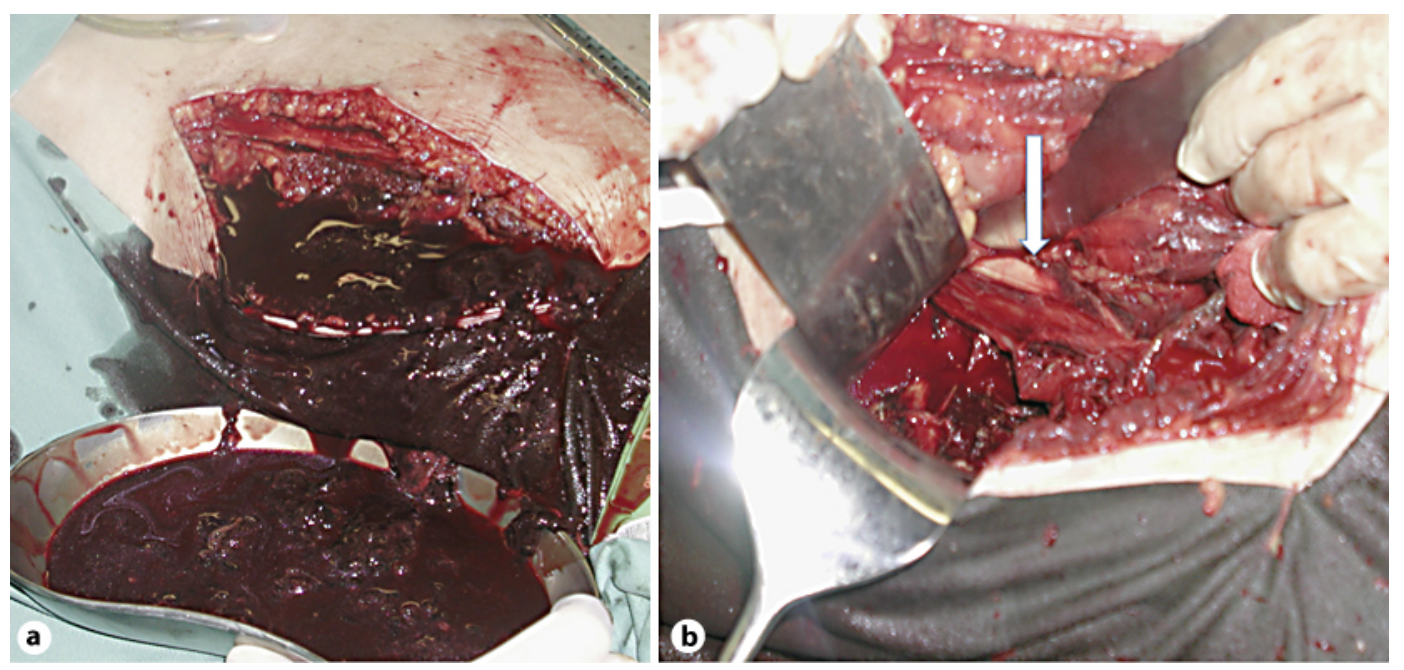

Fig. 2. a, b During laparotomy, a large hematoma and necrotic mass was found in the right psoas muscle (arrow). 


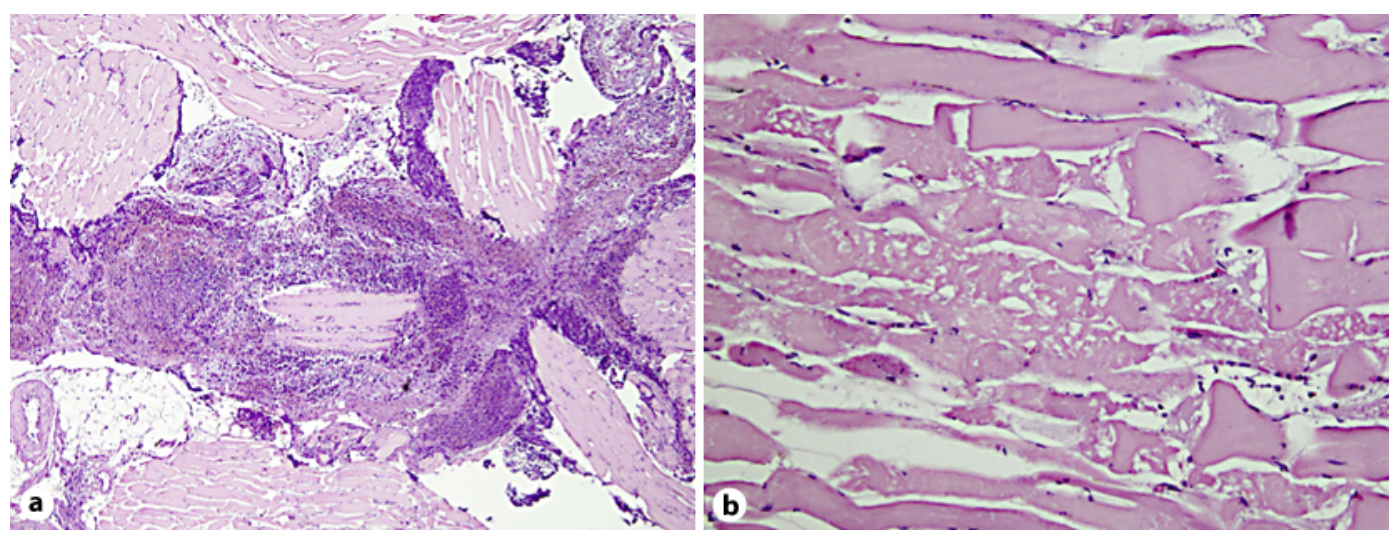

Fig. 3. Histological examination of the resected specimens revealed a psoas muscle with disproportionate fiber sizes and degenerating fibers surrounding an inflammatory reaction; these findings confirmed the diagnosis of fenofibrate-induced rhabdomyolysis (HE stain; $\mathbf{a} \times 40 ; \mathbf{b} \times 200$ ).

\section{References}

1 Sauret JM, Marinides G, Wnag GK: Rhabdomyolysis. Am Fam Phys 2002;65:907-912.

2 Jen Sl, Chen JW, Lee WL, et al: Efficacy and safety of fenofibrate or gemfibrozil on serum lipid profiles in Chinese patients with type IIb hyperlipidemia: a single-blind, randomized, and cross-over study. Chinese Med J 1997;59:217-224.

3 Insua A, Massari F, Rodriguez Moncalvo JJ, et al: Fenofibrate or gemfibozil for treatment of types IIa and IIb primary hyperlipidemia: a randomized, double-blind crossover study. Endocr Pract 2002;8:96-101.

4 Cetinkaya R, Uyanik A, Yildrim R, et al: Fenofibrate monotherapy-induced rhabdomyolysis in a patient with type-2 diabetes. Indian J Med Sci 2008;62:485-459.

$5 \mathrm{Wu}$, Song Y, Li H, et al: Rhabdomyolysis associated with fibrate therapy: review of 76 published cases and a new case report. Eur J Cli Pharmac 2009;65:1169-1174.

6 Graham DJ, Staffa JA, Shatin D, et al: Incidence of hospitalized rhabomyolysis in patients treated with lipid-lowering drugs. JAMA 2004;292:2585-2590.

$\checkmark 7$ Maiguma T, Fujisaki K, Itoh Y, et al: Cell-specific toxicity of fibrates in human embryonal rhabdomyosarcoma cells. Naunyn Schmiedebergs Arch Pharmacol 2003;367:289-296.

8 Davidson MH, Armani A, McKenney JM, et al: Safety considerations with fibrate therapy. Am J Cardiol 2007;99:3C-18C.

-9aida H, Ishibashi M, Nishida H, et al: Rhabdomyolysis induced by psychotropic drugs. Clin Nucl Med 2005;30:569-570.

10 Gordon JK, Magid SK, Maki RG, et al: Elevation of creatine kinase in patients treated with imatinib mesylate (Gleevec $^{\mathrm{TM}}$ ). Leukemia Res 2010;34:827-829.

11 Shimazaki C, Ochiai N, Uchida R, et al: Intramusclar edema as a complication of treatment with imatinib. Leukemia 2003;17:804-810.

12 Penel N, Blay JY, Adenis A, et al: A imatinib as possible cause of severe rhabdomyolysis. N Engl J Med 2008;358:2746-2747.

13 Cohen MH, Williams G, Johnson JR, et al: Approval summary for imatinib mesylate capsules in the treatment of chronic myelogenous leukemia. Clin Cancer 2002;8:935-942.

14 Vanholder R, Sever MS, Erek E, et al: Rhabdomyolysis. J Am Soc Nephrol 2000;11:1553-1561.

15 Danis R, Akbulut A, Ozemen A, et al: Rhabdomyolysis-induced acute renal failure following fenofibrate therapy: a case report and literature review. Case Report Med 2010;2010:1687-1689. 